\title{
Zinc linked with osteoarthritis
}

Osteoarthritis (OA) is

characterized by cartilage

degeneration and new research

published in Cell places the

molecular events that occur during

zinc $\left(\mathrm{Zn}^{2+}\right)$ homeostasis in cells as

key factors in OA pathogenesis.

Could zinc be the missing link in the quest for disease-modifying therapies for OA?

"The association of $\mathrm{Zn}^{2+}$ with OA pathogenesis has been broadly appreciated in the context of its role as a structural component of matrix-degrading enzymes, required for the maturation and activation of these enzymes," explains author Jang-Soo Chun, "but no evidence available to date clearly indicated that $\mathrm{Zn}^{2+}$ had a causal role in OA." In their new study, Chun and colleagues used a series of in vitro and in vivo experiments to determine the role of $\mathrm{Zn}^{2+}$ during cartilage degeneration in $\mathrm{OA}$.

The researchers found that ZIP8, a $\mathrm{Zn}^{2+}$ importer, was upregulated in chondrocytes of OA cartilage from humans and mouse models, which led to increased intracellular $\mathrm{Zn}^{2+}$ levels. This influx caused increased in vitro expression of matrixdegrading enzymes that are crucial factors in OA cartilage destruction, including matrix metalloproteinase (MMP)3, MMP9, MMP12, MMP13 and ADAMTS5.

Next, a series of genetic experiments in mice confirmed the role of Zip8 in OA pathogenesis. Intra-articular injection of an adenoviral vector expressing Zip8 into mice resulted in Zip8 overexpression in cartilage, meniscus, ligament and the synovium. Increased levels of $\mathrm{Zn}^{2+}$, Mmp3 and Mmp13 in conjunction with cartilage destruction were observed 3 weeks after this injection; at 8 weeks, more severe cartilage destruction was observed, alongside osteophyte development and subchondral bone sclerosis. Similar cartilage destruction and subchondral bone sclerosis was observed in transgenic mice

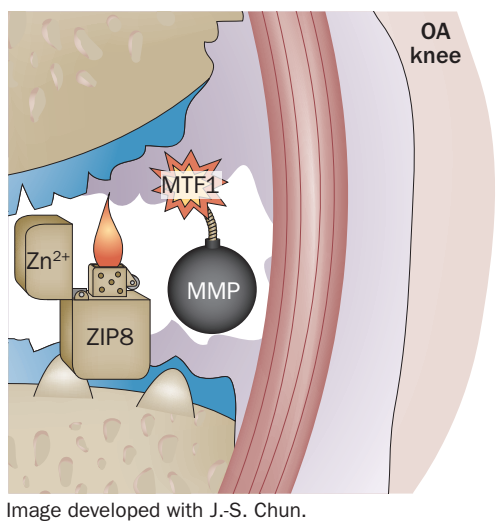

overexpressing Zip8 specifically in chondrocytes. Conversely, experimental OA was inhibited in Zip8-knockout mice; cartilage destruction was notably reduced upon surgical induction of OA, which coincided with inhibition of $\mathrm{Zn}^{2+}$ influx and MMP expression in cartilage tissue.

The investigators identified metal regulator transcription factor 1 (MTF1) as a crucial downstream mediator of the $\mathrm{Zn}^{2+}-\mathrm{ZIP} 8$ activity that fuels the development of cartilage destruction in OA. Importantly, cartilage destruction was substantially reduced in $M t f 1$ knockout mice after surgery to induce OA, whereas deletion of genes encoding metallothioneins, well-known targets of MTF1 that act as $\mathrm{Zn}^{2+}$-storage proteins, led to enhanced OA cartilage destruction in OA mouse models.

"Development of therapeutic antibodies or pharmacological inhibitors targeting the zincZIP8-MTF1 axis can potentially lead to the discovery of a diseasemodifying OA drug," says Chun, "ZIP8 may represent a potentially ideal therapeutic target for treating OA due to its primary localization at the plasma membrane and its specific expression pattern in OA conditions."

\section{Katrina Ray}

Original article Kim, J.-H. et al. Regulation of the catabolic cascade in osteoarthritis by the Zinc-ZIP8-MTF1 axis. Cell 156, 730-743 (2014) 